\title{
Discussion
}

\section{Alzheimer Research Forum Live Discussion: Lost in Translation? Charting the Course in Preclinical Therapeutic Development ${ }^{1}$}

\author{
http://www.alzforum.org/res/for/journal/transcript.asp?liveID=38
}

A panel discussion with Howard Fillit, Jordan Tang, Frank Longo, Eli Michaelis, and Jens Eckstein on 16 September 2005.

Participants: Gabrielle Strobel (Alzheimer Research Forum), Patricia C. Heyn (University of Colorado Health Sciences Center), Jan Teller (Dystonia Medical Research Foundation), Howard Fillit, MD (Institute for the Study of Aging), Kelly R Koehn (Neuroxyn Pharmaceuticals), Jens Eckstein (principal at TVM, a transatlantic VC firm), Cynthia Joyce (Spinal Muscular Atrophy Foundation), Frank Longo (Department of Neurology, University of North Carolina-Chapel Hill), Eli Michaelis (University of Kansas), Jordan Tang (University of Oklahoma Health Science Center), Greg Brewer (Southern Illinois University School of Medicine), Edward Zamrini (University of Alabama Alzheimer's Disease Research Center), June Kinoshita (Alzheimer Research Forum).

Gabrielle Strobel: I am Gabrielle Strobel, managing editor of the Alzheimer Research Forum. I will moderate today. Let me welcome all of you to our discussion. Let us perhaps start by giving our guests a chance to ask their questions first. Where can our panelists help?

Jan Teller: You may ask: What is a dystonia guy doing here? Well, for the last 10 years I have been studying $\mathrm{AD}$, but recently joined the Dystonia Medical Research Foundation (DMRF). So, how can a small foundation like DMRF get creatively and effectively involved in drug development?

Gabrielle Strobel: Jan, you are most welcome here. I suspect some of the issues are similar between $\mathrm{AD}$ and dystonias.

\footnotetext{
${ }^{1}$ Note: The transcript has been edited for clarity and accuracy.
}

Jens Eckstein: Here is one question I have: Where is the bottleneck, actually? Is it novel targets, clinical models, or financing? Any opinions?

Howard Fillit: I think there has been an explosion in novel targets during the past 5 years in Alzheimer's disease (AD). There has also been good progress in animal model development. Financing remains a key issue, in my opinion.

Frank Longo: I think that many potential targets get identified in various in vitro studies. One bottleneck is the ability to move into animal-based models. Components of the bottleneck include access to models, appropriately designed studies, selecting reliable outcomes, etc.

Jordan Tang: I agree that finance is one of the bottlenecks. Many of the targets are untried and represent a high risk. 
Howard Fillit: I agree that access to models in AD is a problem, too. The models remain expensive, and some are limited by intellectual property and licensing issues. I wonder if that is as true in other disease states.

Eli Michaelis: Jens, I agree with Howard that the difficulty is not in identifying new targets but in moving to studies with good animal models and conducting the appropriate pharmacokinetic as well as pharmacodynamic studies. If the work is not done correctly, it would be difficult to raise money to move further along the chain of funding.

Cynthia Joyce: We would like to learn about new ideas for managing intellectual property (IP) transfer from academia to industry. Our foundation is trying to recruit industry to the drug development effort for spinal muscular atrophy (SMA), and the technology transfer process is consuming an inordinate amount of time.

Jens Eckstein: From a venture capital point of view, the less bureaucratic tech transfer is, the better. Technology licensing offices (TLOs) have a pretty bad reputation in the industry and are perceived more as a nuisance. One reason for this is that a lot of them have the mandate from the universities to raise funds rather than get technology out the door.

Cynthia Joyce: That has been our experience. This is a significant bottleneck to doing the pharmacokinetic and pharmacodynamic studies you are discussing. Is there any movement on reducing the bottleneck on licensing models for drug testing?

Jens Eckstein: When talking about targets, one of the key questions remains validation - clinical validation, that is.

Eli Michaelis: Jens, clinical validation could benefit from a universal acceptance of surrogate markers for the disease. This is still lacking in this field.

Gabrielle Strobel: One often reads that the industry over the past 5 years or so has raised its bar on what kind of validation it expects before it considers taking on an academic project. Where is that bar now? What do you have to show?

Jens Eckstein: Validation in our shop means that one sees a clinical effect when hitting the target, that is, Phase 2 data or in some instances, Phase $1 \mathrm{~b}$ data.
Howard Fillit: As Eli points out, while cognition is a "valid" clinical outcome, we do not have valid biological outcomes. That is a big problem for $\mathrm{AD}$ in moving from animals to humans. One unique problem in AD that we always talk about is that "mice do not write books." There was recently a conference on trying to identify cognitive tests that translate well from mice to humans.

Jordan Tang: Animal model work is definitely a slow step, although in working at drug targeting at the initial step of AD pathogenesis, the problem may not be as severe. There are very limited models available for academic laboratories for cognitive tests.

Greg Brewer: It seems that many problems with human trials are neurological issues. Does anyone have an idea how many and how to monitor these better in animal models, for example, pain, blurred vision, confusion?

Eli Michaelis: I agree with Howard that there are improvements being made in behavioral methodology. I would like to comment again on being able to perform good pharmacokinetic and pharmacodynamic studies on normal animals, and animal models of the disease are a prerequisite; otherwise, the results may be too "risky" for a company to invest substantial amounts of money.

Gabrielle Strobel: Eli, how do you recommend an academic investigator - say, a molecular biologist in neuroscience - should approach toxicological and pharmacological testing of a compound he or she considers promising? How does one start this important piece of the package?

Eli Michaelis: Gabrielle, there are a few academic centers that are fully equipped to conduct pharmacokinetic studies and early toxicological studies. There are also several companies that perform these types of studies. What is needed is some early-stage financing that will allow for the conduct of such studies.

Gabrielle Strobel: Eli, is this a niche for foundations?

Eli Michaelis: Gabrielle, yes.

Jens Eckstein: In my opinion, it would be better to focus on safety parameters in the preclinical studies and then push into humans as quickly as possible. New 
biomarkers might make this move more feasible in the future, since one could get surrogate readouts earlier in clinical development.

Gabrielle Strobel: Amyloid imaging biomarkers are being tested in humans, but they are based on positron emission tomography (PET) and are expensive.

Howard Fillit: I personally "believe" PET amyloid imaging could change the paradigm, at least for targets on amyloid. In addition, similar imaging modalities for tangles or neuronal markers are on the horizon. Here is a factoid to consider that I think is still reasonably true. Of about 250 drugs approved by the Food and Drug Administration (FDA) in the past 5 years, only around 10 were for central nervous system (CNS) diseases, all symptomatic therapies, and none were disease-modifying, so it is not just $\mathrm{AD}$, but neurological disease in general.

Jan Teller: Howard, what, in your opinion, is the reason for so few CNS drugs approved by the FDA?

Howard Fillit: It is not the approval process; it is the ability of science/pharma/biotechs to deliver agents for approval to the FDA. These are difficult diseases and targets. I think outcome measures are secondary issues.

Jordan Tang: For an academic molecular biologist, it would probably take a very serious drug candidate before toxicology and pharmacokinetics can be considered. Besides, these tests are usually more expensive than the grant can support. So, some kind of commercial outfit needs to be organized. These all make it a difficult task.

Frank Longo: A potential important asset might be an National Institutes of Health (NIH)-funded mechanism/service in which a large number of investigators with multiple potential targets could assess compounds in animal models using a critical mass of expertise in trial design, pharmacokinetics, and toxicology. This would also allow some comparative function.

Howard Fillit: Of course, the Institute for the Study of Aging (ISOA) has given about \$24 million to over 130 "high-risk" early-stage drug discovery programs during the past 5 years. We have also supported some pharmacokinetics/pharmacodynamics toxicology, GMP (Good Manufacturing Practice) synthesis, etc. There is a great demand for our funding, and we are trying to raise money to meet this need through a new public charity. The demand from academia and biotech, in my opinion, demonstrates that it is not a lack of ideas; it is a lack of funding.

Jens Eckstein: I agree that independent new drug (IND)-enabling toxicology is usually too expensive for research grants. This is a place the NIH should really focus on since they have the resources as well as the expertise for these studies.

June Kinoshita: Frank seems to have suggested that the NIH can also play a role by funding core centers to provide these services to academics. Do you know of actual moves afoot within NIH to do this?

Howard Fillit: I think the National Institute on Aging (NIA) does have a program for supporting toxicology studies now.

Jordan Tang: The NIH Translational Research Award seems to be designed to do some of the preclinical studies.

Eli Michaelis: Jordan, if a scientist has access to other scientists with expertise in pharmacokinetics and toxicology studies, these early studies can be carried out in academic settings for considerably less money than in industrial settings.

Frank Longo: Another key challenge for many is behavioral and cognitive assessment in animal models. There seem to be some commercial sources, but it is expensive and it is hard to execute animal work off-site.

Jens Eckstein: When working with academic laboratories on IND-enabling studies, it is very important to include someone who has done INDs and clinical development. There are a lot of tricks and pitfalls. Pharma people are a great resource in this respect.

Howard Fillit: Jens, I agree that most academic and some early-stage biotech companies lack expertise in preclinical and clinical development. Partnering is definitely key, and planning needs to start early on.

Frank Longo: Even when one can access the technical capabilities, I have been amazed at how many opinions one can get on the best preclinical, IND-type strategies. It would be nice to have a consulting team to go to for advice. 
Howard Fillit: In response to Frank's comments, the "drug discovery services" industry is now out there. What are people's experiences in consulting with them?

Eli Michaelis: Frank, I agree with you that this is a complex process and nothing is immediately obvious, including the opinions you receive. In our experience, we have found that if we have conducted some good studies of pharmacokinetics and have evidence of good pharmacodynamic properties without obvious short-term toxicology, then pharma companies become interested in co-funding or helping to launch a company for the further testing and development of a drug.

Jens Eckstein: I would also like to point out that VCs (venture capitalist firms) can be a great resource, since they usually view the IND-enabling studies as one of the key components for an investment. They are very well connected and are in the people business.

Jan Teller: Could someone comment on the applicability/validity of non-vertebrate testing in CNS diseases and its current perception by pharma and the FDA?

Frank Longo: The jump from mouse to human has been so difficult; I imagine an even larger jump is more difficult, but on the other hand, the elegance of some of the invertebrate models is impressive.

Eli Michaelis: Jan, non-vertebrate testing is, I believe, still a long way away from being accepted as a substitute to vertebrate testing.

Howard Fillit: Non-vertebrate testing has value for target validation in a biological sphere. But worms do not write books. That is the problem.

Frank Longo: I think Eli is right. One interesting area, though, is the potential ability to screen large numbers of compounds with invertebrate models; thus, what one loses in increased risk of applicability might be gained with the increment in numbers.

Greg Brewer: What are your opinions about getting a pre-IND plan from an FDA consultant versus your own or a consulting firm?

Jens Eckstein: The most important issue when working with contract research organizations (CROs) is project management; one should not rely on their internal expertise, since ultimately they need to earn money and have a kind of "conflict of interest" to do only what is necessary.

Frank Longo: I have had some good experiences in getting specific services; others are too expensive. Getting advice on the big picture requires some networking; I have no experience with the FDA.

Jens Eckstein: Pre-IND meetings are wonderful, although they do now (or early next year?) cost money. It is absolutely essential to establish a personal rapport with the FDA folks working on your IND and clinical trial, and a lot of mistakes and misunderstandings can be cleared away.

Jordan Tang: Our experience is to do all the toxicology and pharmacokinetics within the framework of a new startup. It enabled us to connect with expert service and advice. We would not be able to do it without a new venture.

Jens Eckstein: The problem is that it is very difficult to raise VC money with a new target idea and no clinical data.

June Kinoshita: To all, is drug delivery across the blood-brain barrier (BBB) a major obstacle to CNS drug development? One would think so, but many academic scientists I have spoken with seem to blithely assume that once they have come up with a compound, it is not a big deal for pharma to get it across the BBB. Does the neurodegeneration field (and its funding sources) need a wake-up call on this issue?

Howard Fillit: The BBB is a problem; there is no doubt. But I think we are learning more and more about the chemical properties of compounds that can cross, and how to design them.

Jens Eckstein: The earlier people with novel compounds hook up with formulation/delivery people, the better.

Jordan Tang: For people in pharma who are working on drugs, BBB penetration is a big problem. It requires the design of very small molecules which are not compatible with many of the other good properties for a drug.

Eli Michaelis: June, you are right about the BBB being a true "barrier" to CNS drug development. But, it 
is not true that neuroscientists in the area of drug development have ignored that. I know that our scientists have developed in vitro models of the BBB that are used routinely, early in the drug design process, in order to select the best candidates for further development.

Frank Longo: The BBB problem is critical for VC. The medicinal chemistry costs and the uncertainty of whether a compound can be modified appropriately change the cost/risk equation.

Eli Michaelis: I would recommend to all to pay very close attention to the brain delivery issue. Big pharma has learned that lesson very well. Most failures in drug development have been associated with problems in delivery.

Jordan Tang: BBB is just one of the many problems. Selectivity is also a critical problem and, previously mentioned, toxicology and pharmacokinetics. Then there is oral availability. The problem is how to incorporate all these good properties in a small molecule.

Frank Longo: We recently had a series of compounds assessed commercially for BBB penetration in vivo in mice. The cost was about $\$ 10,000$ for each compound.

Howard Fillit: We have certainly seen programs in which animal model studies are proposed, without data on whether the compounds get into the brain. That is bad.

Frank Longo: It is expensive to obtain measurements of compounds in the CNS; thus, I imagine there are many unpublished studies of compounds not working in which the CNS bioavailability was not certain.

Eli Michaelis: June, the reason why I mentioned that pharmacokinetics studies should be done early on is so that direct measures of penetration can be made. Preferably, if scientists have access to BBB cell models, they will have determined if drugs do or do not penetrate the $\mathrm{BBB}$ so they do not reach the wrong conclusions.

June Kinoshita: Eli, are such BBB cell models readily available to academic scientists?

Eli Michaelis: June, the models have been around for nearly two decades and can be established in academic laboratories. Our scientists have been conducting workshops for academic and industrial scientists to teach them how to set up these models.
Jens Eckstein: I think that the BBB problem is one piece of the puzzle. As for our shop, we are more concerned about mechanism of action/efficacy than the BBB. In our experience, the formulation/delivery folks can do wonders.

Eli Michaelis: Jens, the issue is not that you would equate rodent to human pharmacokinetics. It is that you would know how to interpret your pharmacodynamic results if you know how the pharmacokinetics profile is in the experimental animal you are using.

Jens Eckstein: Fair enough, Eli. But again, I would circumvent the pharmacodynamic/pharmacokinetics issue as long as possible and show efficacy by direct delivery.

Jordan Tang: We have used the cell model of BBB but still wanted to know how it comes out in an animal model. With the power of mass spectrometry, it is not too difficult to measure drugs in the brain.

Howard Fillit: I agree with Eli; the models have been around a long time, but I do not know about actual data on how predictive they are of clinical success.

Eli Michaelis: Howard, in our experience they are quite accurate, including the prediction of the pathway for the block in BBB transfer.

Howard Fillit: Another approach is using PET in early-stage clinical studies with labeled drug. I have seen that done.

Jens Eckstein: PET is unfortunately very expensive and clinicians have been very slow in adopting PET in clinical trials.

Howard Fillit: For AD, if the target is amyloid, I would prefer to see a change in amyloid deposition, for example. Rodent cognition testing is very poorly predictive of human success, as I understand the data.

Gabrielle Strobel: Howard, that is interesting. Can you elaborate? Do you mean Pittsburgh compound B (PIB) being used in AD trials?

Howard Fillit: PIB is good for amyloid imaging, but if one is trying to expedite pharmacokinetics/pharmacodynamics in humans, in CNS, PET may be useful with some drugs that, for example, affect 
metabolism neurochemistry. That is, distinguish PIB as a biomarker study from the issues around; does a drug get into human brain and where in brain does it go?

Frank Longo: I think that significant sensitivity and specificity issues remain with PET, even with the labeled compounds that bind amyloid.

Jens Eckstein: I should add that PET is especially expensive in the US. In Australia or New Zealand, PET costs a third or even a fourth of the US price tag.

Gabrielle Strobel: One Australian center reported initial results of exploring the use of PIB in AD and related dementias at a conference earlier this year.

Greg Brewer: Given the BBB problem, is there any receptivity to craniotomy and direct injection or longterm pumping for chronic problems?

Howard Fillit: I do not think craniotomy is a promising way to go, or direct injection. But I think intranasal delivery looks very promising, especially new work in the last couple of years on this delivery method.

Eli Michaelis: Greg, if that is the only way to get it in there and it is a serious disease, maybe. However, I agree with Howard about the intranasal route.

Howard Fillit: There is hope for nanotechnology in delivery across the BBB.

Jens Eckstein: Intranasal delivery really has huge potential.

June Kinoshita: Do you think some promising compounds are being written off too soon because animal studies do not properly take into consideration the formulation and delivery issue? That is, do you worry that inattention or lack of experience to drug formulation and delivery could lead to false negative results in animal studies?

Jens Eckstein: June, that might be true; however, it is dangerous to optimize compounds toward rodent pharmacokinetics.

June Kinoshita: Jens, can you elaborate on your comment?
Jens Eckstein: Rodent pharmacokinetics can be very different from human pharmacokinetics, and you could have spent a lot of time and money to get it right with mice only to find out that the pharmacokinetics profile does not work for humans. If one wants to show the drug works, then go the intracranial route and do not worry too much about the pharmacokinetics in mice.

Gabrielle Strobel: Jens, there are a few vaccine approaches in AD that use the nasal route. Will drugs that come in through the nasal epithelium spread far enough in the brain, though?

Howard Fillit: Gabrielle, there are two wellestablished routes of delivery by intranasal method: through olfactory and trigeminal nerve pathways. Both allow rapid delivery to the entire brain through specific routes. These have been worked out recently. Think about cocaine: It gets almost immediately to the hippocampus.

Gabrielle Strobel: Howard, does this apply equally to small molecules like cocaine and larger molecules like proteins, that is, an immunization antigen?

Howard Fillit: The advantage of intranasal delivery, by the way, is fewer systemic side effects. And yes, peptides are fairly rapidly transported to the brain. Vaccination is a different matter; that actually happens in the intranasal epithelium, not the brain.

Frank Longo: Assuming one gets the compound in the brain adequately, which is more important in terms of an early, efficient milestone? Cognitive effects, morphology, other endpoints? With limited funding, what area should be prioritized early?

Eli Michaelis: Frank, you are raising one of the most difficult issues in experimental design of treatment approaches. If you treat till you see a change in behavior or neurological signs, you may have allowed neurodegeneration to go on too long to see possible amelioration of the path at an earlier stage.

Frank Longo: Eli, I have the same sense you do. If one argues that synaptic function is one of the first functions to lose, though, perhaps cognitive testing would pick up an effect prior to looking at path changes, etc. I think it depends on which model and the mechanism of the compound being tested.

Eli Michaelis: Frank, I agree. 
Howard Fillit: In humans, cognitive testing is ultimately key, but for proof of concept in animals, I think the biological target is more important.

Gabrielle Strobel: Eli, is there any practical way to measure synaptic function instead of behavior/ neurologic function?

Eli Michaelis: Gabrielle, there are attempts being made to label neurons in vivo with fluorescent or chemiluminescent dyes and study dynamic changes with surface, highly sensitive cameras. This is an evolving technology and is not yet ready for direct assessment of synaptic function at this point in time.

Howard Fillit: I think the only way to look at synapse function now is at autopsy of the animals.

Greg Brewer: Synaptic function is most often assessed in vitro with isolated cultures or slices of rat hippocampal tissue.

Eli Michaelis: Howard and Greg, you are both right, but that terminates the ongoing study; that is, it becomes a single time point.

Edward Zamrini: We still do not know if we need to target amyloid deposition (bad or good because it gets rid of soluble amyloid) or soluble amyloid molecules.

Jordan Tang: Has there been a prediction model on the relationship of amyloid lowering and cognitive improvement?

Frank Longo: Jordan, I do not know of clear correlation between amyloid lowering and cognitive improvement clinically. It is also interesting to think about potential physiological functions of $\mathrm{A} \beta$ and recent data suggesting that lowering it too much might be a limiting factor.

June Kinoshita: Frank, to which study are you referring?

Frank Longo: There was an in vitro study by Plant et al. [1] showing that removing $\mathrm{A} \beta$ was deleterious in the BACE knockout $(\mathrm{KO})$ mice in which Y-maze performance was impaired.

Greg Brewer: Jordan, the behavior studies with mice together with the amyloid lowering data are what gave
Elan enthusiasm to go to clinical trials with antiamyloid immunization.

Howard Fillit: Greg is right on that. It is a good example.

Greg Brewer: Frank, Jordan, the clinical data from the Elan Phase 2 trial showed some cases of lower plaque load postmortem, but the cognitive scores were not remarkable, in my opinion.

Jan Teller: Jordan, both animal and human studies suggest that one has to lower soluble amyloid, but what is soluble $\mathrm{A} \beta$ in the brain? What you define as soluble is after extraction from the brain.

Edward Zamrini: Historically, clinical trials have focused on cognitive testing. However, as we advance to neuroprotective therapies, we will need to rely on neuroimaging or biological markers, as neuropsychological markers will not be sensitive enough.

Howard Fillit: I am not sure I agree totally with you, Edward. I think the primary outcome will always be cognition because dementia is a disease of cognition, but to get an FDA approval for a disease-modifying agent will require biological marker studies as well.

Edward Zamrini: Howard, I agree with you when we speak of dementia/mild cognitive impairment (MCI). However, at some point, we will want to do prevention studies. Unless we have appropriate biomarkers that can detect subtle disease (or disease risk) changes, we will not be able to test enough compounds in enough people in a reasonable period of time.

Frank Longo: I think "biomarkers" is an attractive, powerful term; in the end, cognitive function might be the ultimate biomarker.

Jens Eckstein: Is anybody actually looking for a biomarker for "cognition"?

Howard Fillit: Greg points out an important problem in clinical trials to date: The effect size has been small for almost all therapies. I am not sure if this is because, once you have lost neurons, you remain demented and that is it, or if the drugs we have tested thus far just are not good enough.

Gabrielle Strobel: The biomarker issue may simply take more time, as natural history studies track pro- 
gression along with changes in blood and cerebrospinal fluid (CSF) samples. Such a study is underway.

Eli Michaelis: Jens, I thought that is what the synaptic integrity and synaptic function assessment was designed to be.

Howard Fillit: I also agree with Frank completely that cognition is a good clinical biomarker, but the FDA wants evidence of an alteration in the disease brain (read, a change in a traditional biological biomarker).

Jens Eckstein: Howard, what is the FDA's argument here? That the cognition endpoints are too soft?

Howard Fillit: The recent paradigm of "prevention trials" has been delaying MCI to dementia. But preventing dementia has also been demonstrated in hypertension trials. Essentially, the answer to your question, Jens, is yes, but I do not think it is just "bias" on cognitive measures, or softness; it is that cognition is a symptom. To get a labeling for a disease-modifying agent requires biological plausibility that the agent modifies something (read, plausible mechanism of action).

Jan Teller: Instead of talking about cognition in general, should we talk about specific cognitive tasks that can be measured and, therefore, become biomarkers?

Frank Longo: Array/proteomic approaches are already influencing breast cancer trials; there is an example where marker data of a non-traditional type plays a potential clinical role.

Howard Fillit: Proteomic markers are certainly being studied in AD. But I still do not think even specific cognitive tasks will ultimately allow for a diseasemodifying label from the FDA.

Eli Michaelis: Howard, are these proteomic targets outside the CNS or in the CNS?

Howard Fillit: Proteomics in CSF or blood.

Edward Zamrini: Helen Kim from the University of Alabama at Birmingham presented proteomic data (animal) at the June Alzheimer's meeting in DC.

June Kinoshita: David Bennett and colleagues at Perkin Elmer also recently published an interesting proteomic study of AD biomarkers in serum [2].
Jens Eckstein: The problem is that adoption of array/proteomic data in the clinic is terribly slow. It took oncologists more than 15 years to check on p53 status.

June Kinoshita: To all, we are nearing the end of the hour. Clearly, there are daunting challenges for anyone - academic, biotech, or pharma - trying to develop treatments for AD. But there are also untapped opportunities and insights that could help accelerate early-stage work, especially for academics. Could each of our invited speakers summarize the most useful take-home message for scientists who are "lost in translation"?

Jordan Tang: If the majority of AD cases are associated with the elevation of $\mathrm{A} \beta$ (especially 42 ) in the brain, then it would be useful to have a way of measuring "soluble" amyloid in the brain. Obviously, this is difficult and has not been accomplished.

Howard Fillit: My advice to academics is to emulate Eli Michaelis at the University of Kansas and the multidisciplinary teams and centers built to do drug discovery. Traditional biology mechanism of action research programs cannot do it alone.

Frank Longo: Take-home message: Get lots of input from multiple academic and industry individuals who have experience with thinking in terms of milestones and valid targets. I agree the University of Kansas model is a nice academic/industry-type expertise blend.

June Kinoshita: It has become rather fashionable now for big university medical schools to set up drug discovery centers. Any opinions on which ones (besides Eli's) are successful models? What pitfalls should they watch for?

Howard Fillit: The Laboratory for Drug Discovery in Neurodegeneration (LDDN) at Harvard is another reasonable model.

Jens Eckstein: One major shortcoming of these centers is the lack of process development and formulation/delivery folks.

Howard Fillit: Jens, I agree, but that can be outsourced. Also, the emerging regional biotech centers are trying to build these capabilities.

Jordan Tang: The LDDN at Harvard is a great model for academia centers. A lot of good data can be pro- 
duced to show feasibility that would make the project much more attractive to venture funds.

Eli Michaelis: June, you are right that it is difficult to bring together the expertise of a pharma company in a single center, but it has to be done if it is going to succeed. You need more than good biology and chemistry; you need a good understanding of pharmaceutics and a know-how of what industry faces in drug development.

Jens Eckstein: Outsourcing means good project management; that is, no matter what you do, you want to have people in the effort with expertise in process development/formulation/delivery.
June Kinoshita: Thank you, all. This has been a terrific discussion.

\section{References}

[1] L.D. Plant, J.P. Boyle, I.F. Smith, C. Peers and H.A. Pearson, The production of amyloid beta peptide is a critical requirement for the viability of central neurons, J Neurosci 23 (2003), 55315535.

[2] M.F. Lopez, A. Mikulskis, S. Kuzdzal, D.A. Bennett, J. Kelly, E. Golenko, J. Dicesare, E. Denoyer, W.F. Patton, R. Ediger, L. Sapp, T. Ziegert, C. Lynch, S. Kramer, G.R. Whiteley, M.R. Wall, D.P. Mannion, G. Della Cioppa, J.S. Rakitan and G.M. Wolfe, High-resolution serum proteomic profiling of Alzheimer's disease samples reveals disease-specific, carrierprotein-bound mass signatures, Clin Chem 51 (2005), 19461954. 